\title{
Long-term outcomes of direct percutaneous endoscopic jejunostomy: a 10-year cohort
}

Authors

Institutions
Amanda H. Lim ${ }^{1}$, Mark N. Schoeman ${ }^{1}$, Nam Q. Nguyen ${ }^{1,2}$

${ }^{1}$ Departments of Gastroenterology and Hepatology, Royal Adelaide Hospital, Adelaide, Australia

${ }^{2}$ University Department of Medicine, University of Adelaide, Royal Adelaide Hospital, Adelaide, Australia submitted:

10. December 2014 accepted after revision: 13. July 2015

\section{Bibliography}

DOI http://dx.doi.org/

10.1055/s-0034-1392806

Published online: 15.9.2015

Endoscopy International Open

2015; 03: E610-E614

(c) Georg Thieme Verlag KG

Stuttgart · New York

E-ISSN 2196-9736

\section{Corresponding author}

Nam Q. Nguyen, PhD

Department of

Gastroenterology and

Hepatology

Royal Adelaide Hospital

North Terrace

Adelaide

South Australia 5000

Australia

Fax: +61-8-82235885

quoc.nguyen@health.sa.gov.au
Study aim: To assess the clinical outcomes of patients who received direct percutaneous endoscopic jejunostomy (DPEJ) for enteral feeding. Materials and methods: This is a 10-year cohort study in a single tertiary center. Main outcome measurements were technical success, and shortand long-term outcomes. DPEJ was attempted in 83 patients ( 51 men; $55 \pm 2$ years) for dysphagia $(n=35)$, gastroparesis with recurrent aspiration $(\mathrm{n}=30)$, and levodopa drug infusion for severe Parkinson's disease $(n=18)$.

Results: DPEJ was successful in 75 (90\%) patients. All technical failures were related to the inability to find adequate trans-illumination, and were not influenced by BMI, age, gender, or indication. Peri-operative (30-day) adverse events occurred in $11(13 \%)$ patients, including wound infection (3), leakage around the stoma (4), minor bleeding requiring no intervention (2), and aspiration (1). There was one case $(1.2 \%)$ of gastric perforation after PEJ insertion for levodopa drug infusion trial. This 60-year-old woman required an emergency laparotomy with nil complications, and levodopa drug infusion recommenced successfully. One

\section{Introduction}

$\nabla$

Enteral feeding is a critical component of nutritional support in individuals who cannot tolerate adequate oral intake. In patients with a functioning gut, enteral feeding is preferred to parenteral feeding [1-3]. Short-term enteral feeding tubes are placed when such devices are expected to be in situ for less than 30 days duration [4]. For those likely to require long-term feeding (longer than 30 days), multiple options exist including percutaneous endoscopic gastrostomy (PEG), PEG with a jejunal extension tube (PEGJ), surgical jejunostomy (SJ) or direct percutaneous endoscopic jejunostomy (DPEJ). Enteral nutritional support via PEG is often considered to be the first-line approach as PEG insertion is technically easy and case of intestinal perforation (1.2\%) occurred after jejunostomy tube replacement at 6 months of insertion, which was successfully managed with surgery. There were no peri-operative deaths. Adequate delivery of enteral feeding or Duodopa drug was achieved in 66/73 (90\%) patients, with evidence of weight gain or improvement in Parkinson's disease. Seven (8\%) continued to have clinical regurgitation but not aspiration. After a median follow-up of 84 months, 27 (33\%) patients died of their underlying diseases. Seven $(8 \%)$ had marked improvement in their underlying disease and had PEJ removed after 5 months (range $1-8$ months).

Limitations: Single center study.

Conclusions: DPEJ is associated with a high technical success rate (90\%), a relatively low rate of peri-operative adverse events (13\%) and an improvement in long-term nutritional support in the majority of patients (90\%). DPEJ should be the procedure of choice to gain enteral access for feeding or drug delivery prior to considering surgery.

the procedure can be done by most endoscopists. However, gastric feeding via PEG is not suitable for all patients, especially those whose gastric function is impaired (i.e. gastroparesis) or those who are at risk of aspiration pneumonitis [1-3]. In these cases, delivery of nutrients directly to the small intestine is a preferred approach. In addition, DPEJ is able to overcome the frequent tube clogging or obstruction of the thin jejunal extension tube of PEGJ, providing a more secure and sustained feeding approach. In cases of severe gastroparesis or partial gastric outlet obstruction, the use of DPEJ also allows insertion of a concurrent $P E G$ for venting purposes.

First described by Shike et al. in 1987, DPEJ involves the direct insertion of a feeding tube into the proximal small intestine via endoscopic tech- 

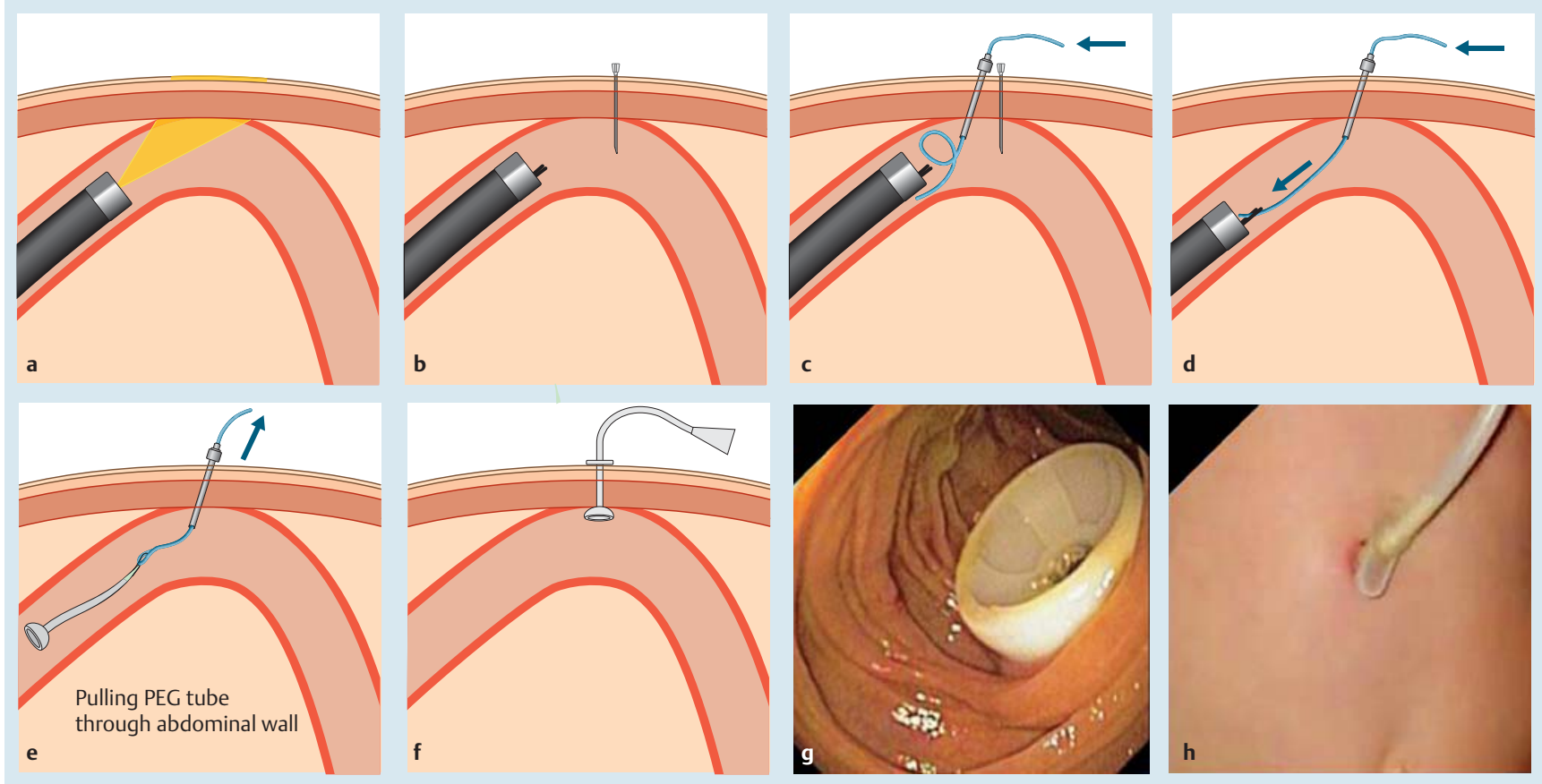

Fig. 1 Outline of steps involved in the insertion of DPEJ. a Identification of good light trans-illumination once the scope reaches the jejunum. $\mathbf{b}$ The identified loop is quickly fixed using a $22 \mathrm{G}$ venesection needle. $\mathrm{c}$ The trocar needle is then inserted along the side of the fixing $22 \mathrm{G}$ needle and the wire is passed through the trocar needle into the intestinal lumen. $\mathbf{d}$ The wire is snared and pulled out of the mouth by pulling both the scope and snare out of the patient. $\mathbf{e}$ The 20F traction-removable PEG tube is connected to the wire and the PEG is pulled through the jejunal loop and out of the abdominal wall. $\mathbf{f}$ The PEG tube is then fixed to the abdominal wall. $\mathbf{g}$ The internal bumper of the PEG. $\mathbf{h}$ The external feeding tube of the DPEJ.

niques [5]. This modality has gained increasing popularity over the last decade, and provides adequate enteral nutritional support without requiring surgery. Compared to PEGJ, DPEJ has been shown to have lower rates of reintervention ( $31 \%$ vs. $75 \%$ ) [6]. Short-term data have also demonstrated that DPEJ increases body weight and reduces recurrent aspiration pneumonia in patients with a history of aspiration pneumonia [7,8]. To date, little data currently exist with regard to the long-term clinical outcomes with DPEJ. This study aimed to assess the technical success, complications, and long-term clinical outcomes in patients who received a DPEJ.

\section{Materials and methods \\ $\nabla$}

All medical, endoscopic, and surgical records related to all patients who were referred for DPEJ insertion between 2003 and 2013 at the Gastrointestinal Investigation Unit of the Royal Adelaide Hospital, were prospectively collected. Relevant data that were collected included: demographics, body mass index (BMI), comorbidities, indications for DPEJ, procedural success, basic bloods, nutritional status, and progress. The outcomes measured were rates of technical success, short- and long-term complications as well as long-term clinical effects. For each patient, written consent either from the patient or from the legal guardian of the patient was obtained for the procedure. The study was approved by the Human Research Ethics Committee of the Royal Adelaide Hospital. All authors had access to the study data, and reviewed and approved the final manuscript.

Exclusion criteria for DPEJ placement included ascites, peritonitis, peritoneal carcinomatosis, coagulation disorders (prothrombin time of less than $50 \%$; thrombocytes less than $50 \times 10^{9} / \mathrm{L}$ ), previous complex upper gastrointestinal surgery, previous placement of an anterior abdominal wall mesh, expected duration of jejunal feeding less than 2 months, and inability to give consent.

\section{Technique}

All DPEJs were performed by two experienced interventional endoscopists (MS and NN) using a pediatric colonoscope (Olympus PCF 160AL) and a MIC* Percutaneous Endoscopic Gastrostomy (PEG) kit (20F, Kimberly-Clark, Roswell, GA 30076, USA). All subjects cleansed with local antiseptic and a 22G needle was used to puncture into the lumen of the jejunal loop under direct endoscopic vision ( $\bullet$ Fig. 1 b). This was to temporarily fix and secure the relevant jejunal loop to the abdomen wall, allowing time for injection of local anesthesia (lignocaine $2 \%, 10 \mathrm{~mL}$ ), insertion of a larger bore trocar needle, and a small skin incision ( $\bullet$ Fig. 1 c). A plastic loop-wire was then fed through the trocar needle, which was grasped by a snare and pulled out of the mouth by removing the scope ( $\bullet$ Fig. 1 d). The PEG tube was attached to the loop-wire, pulled through the mouth into the jejunum and out of the abdominal wall at the DPEJ site ( $\bullet$ Fig. $1 \mathbf{e}$ ). The inner bumper was retracted against the anterior abdominal wall ( $\bullet$ Fig. 1f) and fixed by a plastic disc ( $\mathbf{~ F i g . ~} \mathbf{1 g}$ ).

\section{Definitions}

Technical success was defined as the ability to successfully insert a PEG tube into the proximal jejunum, by the techniques described above, and enable water or enteral feed to be delivered into the small intestine $(\boldsymbol{F i g .} \mathbf{1}$ h). Complications were stratified into "major" and "minor", in which "major" adverse events were defined as those that required surgical interventions or led to death. All other adverse events, such as insertion site infection, leakage, fever, abdominal pain or bleeding, were defined as "mi- 
Table 1 Summary of demographics and characteristics of the cohort.

\begin{tabular}{|l|l|}
\hline & $\begin{array}{l}\text { DPEJ cohort } \\
(\mathbf{n = 8 3 )}\end{array}$ \\
\hline Mean age, years & $55 \pm 2$ \\
\hline Gender (male/female) & $51: 32$ \\
\hline Indications for DPEJ & 17 \\
Dysphagia related to gastrointestinal malignancy & 13 \\
Dysphagia related to neuromuscular disease & 5 \\
$\begin{array}{l}\text { Dysphagia from previous upper gastrointestinal surgery } \\
\text { (e. g. fundoplication) }\end{array}$ & 30 \\
Refractory gastroparesis \pm aspiration & 18 \\
$\begin{array}{l}\text { Intrajejunal levodopa infusion for refractory Parkinson's } \\
\text { disease }\end{array}$ & \\
\hline Previous unsuccessful PEG feeding & $29(35 \%)$ \\
\hline Mean body mass index (BMI), kg/m² & $23.8 \pm 0.5$ \\
\hline Mean serum albumin at DPE] insertion, g/L & $29 \pm 1.0$ \\
\hline
\end{tabular}

BMI, body mass index; DPEJ, direct percutaneous endoscopic jejunostomy; PEG, percutaneous endoscopic gastrostomy.

nor". Peristomal infection was defined as the presence of observed local inflammatory signs such as erythema, induration, and exudate with pain or tenderness.

\section{Data analysis}

Most data are expressed as mean \pm standard error of mean (SEM). Data that were not normally distributed are expressed as median and range. Comparison of variables was undertaken using Fisher's exact tests for categorical data, and independent $t$ test or Mann - Whitney test for continuous data sets. Analyses were performed using GraphPad Prism statistical software, version 6 (GraphPad Software Inc., La Jolla, CA, USA). $P<0.05$ was considered to be statistically significant in all analyses.

\section{Results}

$\nabla$

Over 10 years, 83 patients ( 51 men; $55 \pm 2$ years) underwent DPEJ with the following indications: (i) poor or unsafe oral intake due to dysphagia caused by gastrointestinal malignancy $(n=17)$, neuromuscular disease $(n=13)$, previous upper gastrointestinal surgery $(n=5)$; (ii) aspiration and gastroparesis related to neuromuscular diseases $(\mathrm{n}=29)$ and idiopathic condition ( $\mathrm{n}=1)$; and (iii) intrajejunal levodopa infusion for management of severe refractory Parkinson's disease $(n=18)$ ( $\bullet$ Table 1$)$. Twenty-nine (35\%) of these patients had been unsuccessfully fed via a PEG with ongoing feed intolerance and respiratory aspiration. Attempts at postpyloric enteral feeding through a PEG-jejunal extension tube in these patients had also failed due to early tube blockage or retrograde migration of the intestinal tube into the stomach.

\section{Technical success}

Insertion of DPEJ was successful in 75 (90\%) patients, and the major reason for technical failure was the inability to identify transabdominal trans-illumination ( $7 / 8$ failed cases). One unsuccessful attempt was due to altered anatomy with a large hiatus hernia and intrathoracic stomach. Due to the associated multiple comorbidities and high anesthetic risk, only two of eight failed cases were able to undergo surgery for insertion of jejunostomy feeding tube. Overall, there were no significant differences in BMI (21.5 vs. $22.8 \mathrm{~kg} / \mathrm{m}^{2}$ ), age ( 55.0 vs. 53.8 years), gender (male $61 \%$ vs. $50 \%$ ) or indication of DPEJ between successful and unsuccessful cases.

\section{Short-term outcomes}

Peri-operative (30 days) complications occurred in $11(13 \%)$ patients, which included gastric perforation (1), peri-stomal infection (3), leakage around the stoma (4), minor bleeding requiring no intervention (2), and aspiration (1). In all cases, the pain related to the DPEJ insertion settled after 1 week. In the case of gastric perforation, the complication was caused by "piercing the stomach" by the jejunostomy tract, which had been "sandwiched" between the jejunal loop and the abdominal wall. The gastric perforation was closed at laparotomy and the jejunostomy was secured, with levodopa infusion recommencing the next day. There were no procedural or peri-operative related deaths. Six patients (7\%) required DPEJ replacement in less than 6 months due to tube blockage. The mean length of hospital stay for patients who were admitted for the insertion of DPEJ was $4 \pm 1$ days, whereas it was $72 \pm 15$ days for those who were already hospitalized for their underlying conditions.

\section{Long-term outcomes}

Enteral feeding that matched the patient's daily requirement was successfully achieved in $95 \%$ of patients $(n=54 / 57)$ with evidence of $7.9 \pm 4.2 \mathrm{~kg}$ weight gain 3 months after insertion of DPEJ for enteral feeding. Although none of the 30 patients with aspiration due to gastroparesis had further admission due to respiratory aspiration after DPEJ, seven patients continued to report intermittent regurgitation. Intestinal perforation occurred in one patient $(1.2 \%)$ when the jejunostomy tube was replaced at 6 months post-insertion. This was related to performing push enteroscopy to retrieve the broken bumper, which tore the jejunal loop from the tract. This was successfully managed with surgery. In patients who had DPEJ insertion for levodopa drug infusion $(n=18)$ to treat severe Parkinson's disease, all apart from two reported a marked improvement in dyskinesia management. One patient failed to respond to levodopa infusion due to severe refractory dyskinesia related to advanced Parkinson's disease. The other patient suffered from a significant anxiety disorder that resulted in an inability to manage levodopa infusion via the DPEJ, and withdrew from the trial after 1 year of treatment. None of the patients who had the DPEJ insertion for the levodopa infusion had long-term complications.

Overall, the mean duration of tube exchange was $8.2 \pm 2.1$ months; 6 (7\%) patients had a tube blockage and required exchange before 6 months. Over the median follow-up period of 84 months, the mean number of exchanges was $3.4 \pm 1.8$. Seven patients ( $8 \%$ ) showed a marked improvement in their underlying diseases, leading to adequate oral intake and removal of DPEJ after a median duration of 5 months (range: $1-8$ months). Twenty-seven patients $(33 \%$ ) died from their underlying diseases. Survival was similar between patients who had $\mathrm{BMI} \geq 30 \mathrm{~kg} / \mathrm{m}^{2} \mathrm{com}-$ pared to those with $\mathrm{BMI}<30 \mathrm{~kg} / \mathrm{m}^{2}(P=0.78 ; \odot$ Fig. 2$)$.

\section{Discussion}

$\nabla$

This study is one of the largest long-term cohorts to report the outcomes of DPEJ for the delivery of either enteral feeds or medication directly into the proximal intestine for nutritional support or treatment of advanced Parkinson's disease. The main findings of the study are that insertion of DPEJ is: (i) highly feasi- 


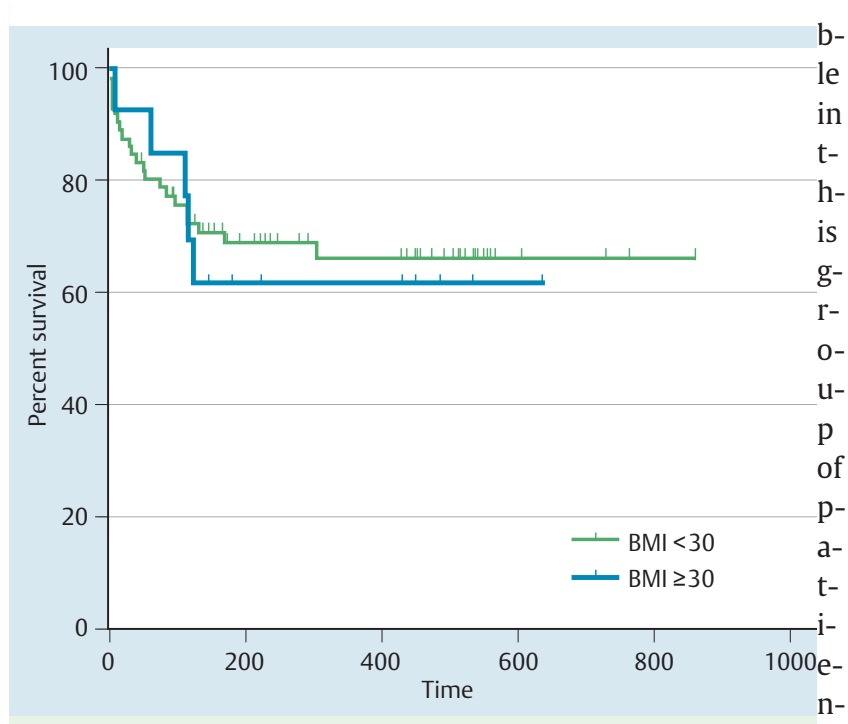

Fig. 2 Kaplan-Meier curves for the survival outcomes of subjects who ts had DPEJ stratified by the presence of obesity $\left(B M I \geq 30 \mathrm{~kg} / \mathrm{m}^{2}\right)$, showing no differences in survival between the groups $(P=0.78)$.

with a $90 \%$ technical success rate, (ii) safe, and (iii) if successful, is associated with improvement in body weight or dyskinesia related to Parkinson's disease. Due to the related comorbidities, approximately one-third of these patients had died after a median follow-up of 84 months. Thus, given the lesser degree of invasiveness compared to surgery, DPEJ should be considered first for patients who require direct access to the small intestinal for nutritional support or drug delivery.

Compared to previous reports [9-13], which had technical success ranging from $68 \%$ to $95 \%$, our study achieved a relatively high technical success with DPEJ insertion (90\%). In contrast to the study by Maple et al. in 2005 [11], which found a lower success rate in patients with a BMI $>30 \mathrm{~kg} / \mathrm{m}^{2}$, we did not find an adverse impact of BMI, age, gender, or indication on the technical success rate. In fact, successful DPEJ insertion was achieved in all three patients with $\mathrm{BMI}>30 \mathrm{~kg} / \mathrm{m}^{2}$ in our study. Instead, the ability to elicit good light trans-illumination and immediate fingerpressure during enteroscopy are the key determinants of the technical success. As with Maple et al. [11], the use of a $22-25 \mathrm{G}$ venipuncture needle to immediately fix the loop of bowel soon after identifying the trans-illuminated loop of bowel prevents untoward movement of the bowel and improves the success rate. More recently, the use of balloon assisted enteroscopy has been reported to improve the technical success of DPEJ [16-19]. With use of a balloon-assisted overtube, and leaving the overtube in place during the procedure, the technical success rate was demonstrated to be 96\% [16]. Balloon assisted enteroscopy DPEJ is also associated with a low complication rate, with a likely factor being improved visual support [17]. Success rates between single- and double-balloon assisted enteroscopy DPEJ are comparable, however double-balloon enteroscopy may be more efficient for deeper intubation of the small bowel $[17,18]$. These emerging data are promising for providing safer and more efficacious DPEJ procedures for patients requiring enteral feeding.

Overall, the procedure was safe with a low risk of perforation (1.2\%) and only minor adverse events occurring in $12 \%$ of cases, which were mostly related to insertion site infection and leakage around the stoma. Furthermore, the need to change the DPEJ due to tube blockage in the first 6 months of insertion was also rare (7\%). In contrast to Maple et al. [11], the overall rate of serious adverse events in our cohort was significantly lower ( $2.4 \%$ vs. $10 \%)$, with one case of gastric perforation related to DPEJ insertion and a case of intestinal perforation related to the replacement of jejunostomy tube 6 months after insertion. The reasons behind the differences remain unclear, but may be related to the technical skills and experience of the two endoscopists who were dedicated to perform DPEJ for South Australia, of which the Royal Adelaide Hospital provides a service to $\sim 500000$ people. In addition, DPEJ only proceeded if good transillumination or immediate finger-pressure was achieved, this measure perhaps preventing inadvertent puncture of overlying organs or traverse loop(s) of bowel before entering the identified loop of bowel. We also highly recommended against performing the enteroscopy to retrieve a broken bumper or immediately after the jejunostomy tube had been removed as we believe that it is the force during the enteroscopy that tears the jejunostomy tract from the abdominal wall and causes perforation. Lastly, we have excluded patients who were potentially at risk of complications due to their underlying diseases or due to extreme technical difficulty (as documented in our exclusion criteria), making our cohort not truly "unselected", which may explain the lower complication rate.

Most importantly, we have demonstrated that DPEJ leads to a positive long-term outcome, with 95\% achieving adequate enteral feeding as evidenced by weight gain (mean of $7.9 \pm 4.2 \mathrm{~kg}$ ) and increase in albumin and hemoglobin. For patients who had aspiration due to gastroparesis, none had further hospital admissions due to respiratory aspiration after DPEJ, though $23 \%$ of patients continued to report some ongoing regurgitation. In $8 \%$ of patients, there was a marked improvement in the underlying disease after nutritional support, leading to resumption of oral intake and removal of DPEJ. Together, these findings indicate that DPEJ in these difficult patients can not only improve their nutrition support but also reduce the number of hospitalizations due to aspiration pneumonia.

Our study is also the first to report the application of DPEJ as a method of direct drug delivery to improve the management of patients with Parkinson's disease. It is well known that gastric emptying is impaired and erratic in advanced Parkinson's disease, leading to an unpredictable rate of drug absorption and poorly controlled dyskinesia. Direct delivery of a drug, such as levodopa, at a constant rate can avoid such problems, resulting in better control of movement disorders. The therapeutic benefit of this concept has been demonstrated by delivering levodopa through the jejunal extension tube via a PEG (i.e. PEGJ). Unfortunately, the jejunal extension tube is small, easily blocked and requires frequent replacement (ranging from every 1 to 3 months). Insertion of the DPEJ, however, overcomes these issues and as demonstrated in our study, the long-term success of levodopa infusion was $89 \%$ with fewer needs to change the DPEJ (every 6-12 months). Two patients required removal due to anxiety and dyskinesia, respectively; there were no other long-term complications recorded.

Finally, our study also shed light on the natural history of these patients, in which over one-third died from their underlying diseases after a median 84 months follow-up. No death in our cohort was related to the technical complications of DPEJ. Although previous studies have shown a much higher 30-day mortality rate, ranging from $17 \%$ to $35 \%$, our cohort consisted of more patients who had advanced malignancy cases as well as other debilitating comorbidities. We had no 30-day mortality, which is most likely 
related to better patient selection as we do not believe that such unwell patients should be subjected to an invasive procedure for little, if any, clinical benefit. The benefit of DPEJ would be best observed in patients with chronic neurological disorders, whose survival is more likely to be years rather than weeks $[9,14,15]$. There were, however, some limitations specific to this study including its single-center nature as well as the use of only a single cohort with no comparison arms. There was also a selection bias towards good procedural candidates, which may be reflective of and applicable to current clinical practice.

In conclusion, DPEJ should be the procedure of choice to gain enteral access for feeding aimed at artificial nutritional support or drug delivery before considering surgery or PEG-jejunal extension due to its high technical success rate (90\%), low incidence of complications (13\%) with the majority being minor complications managed conservatively, low re-intervention rate, and its positive impact on long-term outcomes.

\section{Competing interests: None}

\section{References}

1 Windsor AC, Kanwar S, Li AG et al. Compared with parenteral nutrition, enteral feeding attenuates the acute phase response and improves disease severity in acute pancreatitis. Gut 1998; 42: $431-435$

2 McClave SA, Chang WK, Dhaliwal $R$ et al. Nutrition support in acute pancreatitis: a systematic review of the literature. JPEN J Parenter Enteral Nutr 2006; 30: 143-156

3 McClave SA, Martindale RG, Vanek VW et al. Guidelines for the provision and assessment of nutrition support therapy in the adult critically ill patient: Society of Critical Care Medicine (SCCM) and American Society for Parenteral and Enteral Nutrition (A.S.P.E.N.). JPEN J Parenter Enteral Nutr 2009; 33: 277-316

4 Kirby DF, Delegge MH, Fleming CR. American Gastroenterological Association technical review on tube feeding for enteral nutrition. Gastroenterology 1995; 108: 1282 - 1301

5 Shike M, Schroy P, Ritchie MA et al. Percutaneous endoscopic jejunostomy in cancer patients with previous gastric resection. Gastrointest Endosc 1987; 33: $372-374$

6 Delegge MH, Ginsberg G, McClave S et al. Randomized prospective comparison of direct percutaneous endoscopic jejunostomy (DPEJ) feeding tube placement versus percutaneous endoscopic gastrostomy feeding tube placement with jejunal extension (PEG-J), for enteral feeding. Gastrointest Endosc 2006; 63: AB160

7 Virnig DJ, Frech EJ, Delegge $M H$ et al. Direct percutaneous endoscopic jejunostomy: a case series in pediatric patients. Gastrointest Endosc 2008; 67: $984-987$

8 Panagiotakis PH, DiSario JA, Hilden $K$ et al. DPEJ tube placement prevents aspiration pneumonia in high-risk patients. Nutr Clin Pract 2008; 23: $172-175$

9 Bueno JT, Schattner MA, Barrera $R$ et al. Endoscopic placement of direct percutaneous jejunostomy tubes in patients with complications after esophagectomy. Gastrointest Endosc 2003; 57: 536-540

10 Cope C, Davis AG, Baum RA et al. Direct percutaneous jejunostomy: techniques and applications - ten years experience. Radiology 1998; 209: $747-754$

11 Maple JT, Petersen BT, Baron TH et al. Direct percutaneous endoscopic jejunostomy: outcomes in 307 consecutive attempts. Am J Gastroenterol 2005; 100: $2681-2688$

12 Shike M, Latkany L, Gerdes $H$ et al. Direct percutaneous endoscopic jejunostomies for enteral feeding. Gastrointest Endosc 1996; 44: $536-$ 540

13 Varadarajulu S, Delegge $M H$. Use of a 19-gauge injection needle as a guide for direct percutaneous endoscopic jejunostomy tube placement. Gastrointest Endosc 2003; 57: 942 - 945

14 Moran GW, Fisher NC. Direct percutaneous endoscopic jejunostomy: High completion rates with selective use of a long drainage access needle. Diagn Ther Endosc 2009; 2009: 520879

15 van Overhagen $H$, Ludviksson MA, Lameris JS et al. US and fluoroscopicguided percutaneous jejunostomy: experience in 49 patients. J Vasc Interv Radiol 2000; 11: $101-106$

16 Velazquez-Avina J, Beyer R, Diaz-Tobar $C$ et al. New method of direct percutaneous endoscopic jejunostomy tube placement using balloonassisted enteroscopy with fluoroscopy. Dig Endosc 2015; 27: 317-322

17 Despott EJ, Gabe S, Tripoli $E$ et al. Enteral access by double-balloon enteroscopy: an alternative method of direct percutaneous endoscopic jejunostomy placement. Dig Dis Sci 2011; 56: 494-498

18 Song LM, Baron TH, Saleem A et al. Double-balloon enteroscopy as a rescue technique for failed direct percutaneous endoscopic jejunostomy when using conventional push enteroscopy. Gastrointest Endosc 2012; 76: 675-679

19 Aktas H, Mensink PB, Kulpers E et al. Single-balloon enteroscopy-assisted direct percutaneous endoscopic jejunostomy. Endoscopy 2012; 44: $210-212$ 